\title{
Towards a unified concept of individualized medicine
}

\author{
"...even with the most sophisticated diagnostics tools and algorithm-based \\ treatments, therapeutic success will be determined, to a large extent, by individual \\ priorities and beliefs shaped in a biographic, social and cultural framework..."
}

\section{KEYWORDS: epidemiology $\approx$ individualized medicine $\approx$ multimorbidity $\approx$ personalized medicine $=$ public health}

\section{Evidence-based medicine \& limitations of guidelines}

In the last century, fundamental developments in the sciences have led to important improvements in differential diagnostics and treatment of many diseases. Evidence-based medicine (EBM) provided a conceptual framework for the extraction and condensation of relevant knowledge from the immense body of data that has increasingly become a challenge to clinicians and scientists. In EBM, randomized controlled trials in defined study populations that applied strict inclusion and exclusion criteria were deemed the best research evidence, and systematic reviews were applied to extract valid, clinically relevant information [1]. Hence, EBM marked a major progress from an individual experience-driven practice of medicine to empirically founded, rational medical decision-making that is research driven. Reasoned EBM is operationalized as diagnostic and therapeutic guidelines for clinical practice, including definition, classification, diagnostics, treatment, management and monitoring of a disease.

\section{"...the guidelines only address an increasingly untypical subgroup of patients."}

Guidelines have significantly enhanced the quality of diagnostic and treatment processes by providing more transparency, objectivity and a better handling of information. One constitutional element of the guidelines is standardization. Minimization of internal bias by, for example, strict selection of patient groups, narrow disease definitions, exclusion of comorbidities and selection of certain age groups or sexes favors reductionist approaches but conflicts with the heterogeneity of real life. Often large segments of the general population and specific patient populations with common medical comorbidities and medications are excluded $[2,3]$. The real patient, however, is characterized by properties beyond his or her particular pathology, most of which can neither be fully assessed in randomized controlled trials nor adequately addressed in guidelines. Hence, the highest level of evidence comes at the cost of reduced generalizability up to an extent where the guidelines only address an increasingly untypical subgroup of patients.

\section{Towards individualized medicine}

The era of genomic analysis has put individual properties at the center of interest. From the study of the structure of the genome, mapping genes and sequencing DNA, the term 'individualized medicine' has emerged to emphasize genetic differences between patients. Pharmacogenomics has become relevant in the search for new biological targets and is used as a tool in the design of new drugs and vaccines [4]. In some areas (HIV/AIDS and oncology) genetic testing has entered routine clinical practice (e.g., a gene-expression test for EGF receptors before therapy with gefinitib or test for the existence of the $H L A-B^{*} 5701$ allele before treatment with abacavir). Presently, the German Association of Research-Based Pharmaceutical Companies (VFA) lists ten drugs that require genetic testing prior to therapeutic use [101]. Specialized molecular diagnostic methods and bioinformatic analysis of genetic polymorphisms increasingly allow for a molecular classification of diseases, assessment of differential disease risks and the development of targeted therapies. However, the first applications are linked to rare diseases. For the most prevalent diseases in the population there is relatively little translation that has been done. Hence, traditional genomics must be extended by innovative biomarker-based '-omics'

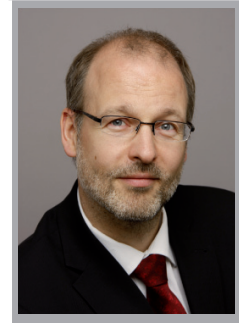

Wolfgang Hoffmann*

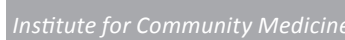
Department Epidemiology of Health Care \& Community Health, Ernst-Moritz-Arndt University of Greifswald, Germany

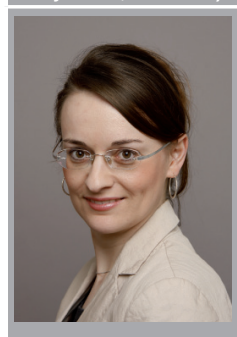

Janina Krafczyk-Korth*

Author for correspondence: Institute for Community Medicine, Department Epidemiology of Health Care \& Community Health, Ernst-Moritz-Arndt University of Greifswald, Ellernholzstr. 1-2, D-17487 Greifswald, Germany Tel.: +493834867789 Fax: +493834867752 janina.krafczyk@uni-greifswald.de Henry Völzke

Institute for Community Medicine, Department Study of Health in Pomerania (SHIP)/ClinicalEpidemiological Research Ernst-Moritz-Arndt University of Greifswald, Germany

Konstanze Fendrich Institute for Community Medicine, Department Epidemiology of Health Care \& Community Health, Ernst-Moritz-Arndt University of Greifswald, Germany

\section{Heyo K Kroemer}

Institute of Pharmacology, Ernst-Moritz-Arndt University of Greifswald, Germany *Authors contributed equally

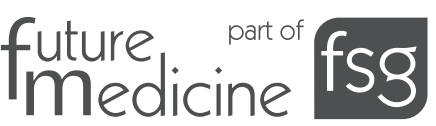


approaches (e.g., transcriptomics, proteomics and metabolomics) and other starting points in individualizing medicine - examples include stem cell therapy in regenerative medicine, individually adjusted prostheses and implants through rapid prototyping, as well as tissue engineering or personal health planning using selfadministered risk algorithms. In a simultaneous bottom-up approach, practical general medicine has pointed out multimorbidity, comorbidity, frailty, functioning and adherence as important individual determinants in modifying response to therapy and outcome. Complex models such as the evidence-based, interdisciplinary and multidimensional chronic care model proposed by Wagner et al. [5] and its extension for prevention and health promotion by Barr et al. were developed to optimize chronic care [6]. Besides emphasizing all of these individual patient factors, these approaches are top-down and to a large extent detached from each other.

"...the concept of individualized medicine requires a holistic and integrative approach."

However, pertinent groups of patients broadly overlap. Given the demographic change and the increase of age-related diseases, multimorbidity increasingly becomes a challenge for all medical specialties. For example, dementia and urinary bladder disease are common comorbidities, but their concomitant treatment may be complicated. While dementia is treated by cholinesterase inhibitors that can trigger incontinence, urine bladder disease is treated by anticholinergic drugs, which can lead to a decline of cerebral function [7]. By designing a common, chronically ill, multimorbid patient and applying the best existing therapeutic guidelines, Boyd et al. demonstrated that adherence to these guidelines can actually be harmful to the patient [8].

Clearly, the concept of individualized medicine requires a holistic and integrative approach. Individualization does not mean abandonment of the philosophy of EBM. However, rather than focusing on the most specific unit of information that is applicable in clinical practice, the guiding evidence needs to be comprehensive and inclusive. Disease-specific guidelines need to consider multimorbidity. In the future, guidelines will no longer be disease-specific, but will target specific combinations of diseases that simultaneously affect the patient with his individual risk factor profile (matrix approach). Diagnostics need to integrate individual genetic and physiological information and, at the same time, consider cognitive, psychological and social resources of the patient. The doctor's task will be to condense all of these aspects into straightforward therapeutic options which can be discussed with the patient. In a shared decision process, it will ultimately be the patient's choice as to what therapeutic goals will be primarily pursued.

\section{Research strategy for the development of individualized medicine}

Presently, medicine is not prepared for this paradigm shift. Intelligent access to basic science data is still limited, and multidimensional algorithms supporting medical decisions have rarely been established in medical practice. To us, the implementation of individualized medicine in routine medical care requires three phases. The first phase is driven by basic, mainly 'omics' research, which will characterize a patient to an almost inconceivable detail. Virtually millions of SNPs, copy number variations, protein modifications and metabolites will be associated with tens of thousands of phenotypes. Functional imaging provides access to subtle subclinical changes as new diagnostic dimensions. Most of this vast body of data will be irrelevant to most patients and most clinical situations. Hence, the first phase will be the search for few needle variables in the haystack of complex information. The criteria to transfer selected variables into the second phase are therapeutic and prognostic relevance. Comprehensive and sustainable biobanking and specialized IT-systems will be essential to integrate the variety of data.

Based on the few informative variables, in the second phase, multidimensional algorithms will be developed. Validity, predictability and clinical relevance of these algorithms will be evaluated in well defined patient cohorts. A fully assessed value of diagnostic and therapeutic algorithms requires extended prospective follow-up and monitoring. To associate the outcomes with the initial algorithm, the integration of regional healthcare provisions are necessary - a prerequisite that will effectively minimize the number of possible study regions in many countries.

In the third phase, validated algorithms will be used to derive evidence-based individualized therapeutic approaches that can be tested in prospective, randomized clinical trials. A successful implementation of theses three phases will move medical care towards a more individualized paradigm. However, even with the most sophisticated diagnostic tools and algorithmbased treatments, therapeutic success will be 
determined, to a large extent, by individual priorities and beliefs shaped in a biographic, social and cultural framework, rendering adherence a result of the often transcultural communication with the physician, blended with gender constructs and charged with a patient's disease concepts and behavior. Hence, the challenge of individualizing medicine will prevail.

\section{Financial \& competing interests disclosure}

This work is part of the research project Greifswald Approach to Individualized Medicine (GANI_MED).

The GANI_MED consortium is funded by the Federal

\section{Bibliography}

1 Sackett DL, Rosenberg WM: The need for evidence-based medicine. J. R. Soc. Med. 88(11), 620-624 (1995).

2 Terschüren C, Gierer S, Brillant C, Paulus U, Löffler M, Hoffmann W: Are patients with Hodgkin lymphoma and high-grade non-Hodgkin lymphoma in clinical therapy optimisation protocols representative for the entire groups of these patients in Germany? Ann. Oncol. 21(10), 2045-2051 (2010).

3 Van Spall HGC, Toren A, Kiss A, Fowler RA: Eligibility criteria of randomized controlled trials published in high-impact general medical journals. A systematic sampling review. JAMA 297(11), 1233-1240 (2007).

Ministry of Education and Research (03IS2061A) and the Ministry of Cultural Affairs of the Federal State of Mecklenburg - West Pomerania. This work was further supported by Siemens Healthcare, Erlangen, Germany. The University of Greifswald is a member of the 'Center of Knowledge Interchange' program of the Siemens $A G$. The authors have no other relevant affliations or financial involvement with any organization or entity with a financial interest in or financial conflict with the subject matter or materials discussed in the manuscript apart from those disclosed.

No writing assistance was utilized in the production of this manuscript.

4 Kroemer HK, Meyer zu Schwabedissen HE: A piece in the puzzle of personalized medicine. Clin. Pharmacol. Ther. 87(1), 19-20 (2010).

5 Wagner EH, Davis C, Schaefer J, von Korff M, Austin B: A survey of leading chronic disease management programs: are they consistent with the literature? Manag. Care Q. 7(3), 56-66 (1999).

6 Barr VJ, Robinson S, Marin-Link B et al.: The expanded chronic care model: an integration of concepts and strategies from population health promotion and the chronic care model. Hosp. Q. 7(1), 73-82 (2003).

7 Sink KM, Thomas J 3rd, Xu H, Craig B, Kritchevsky S, Sands LP: Dual use of bladder anticholinergics and cholinesterase inhibitors: long-term functional and cognitive outcomes. J. Am. Geriatr. Soc. 56(5), 847-853 (2008).
8 Boyd CM, Darer J, Boult C, Fried LP, Boult L, Wu AW: Clinical practice guidelines and quality of care for older patients with multiple comorbid diseases: implications for pay for performance. JAMA 294(6), 716-724 (2005).

\section{Website}

101 German Association of Research-based Pharmaceutical Companies. Berlin, Germany www.vfa.de/de/forschung/am-entwicklung/ individualisierte-medizin.html (Accessed 3 March 2010) 